\begin{tabular}{|c|l|}
\hline Title & $\begin{array}{l}\text { Transient occurrence of vasa expressing cells in nongenital segments during embry onic development in the oligochaete } \\
\text { annelid Tubifex tubifex }\end{array}$ \\
\hline Author(s) & Oyama, A tsuko; Shimizu, Takashi \\
\hline Citation & $\begin{array}{l}\text { Development Genes and Evolution, 217(10), 675-690 } \\
\text { https://doi.org/10.1007/300427-007-0180-1 }\end{array}$ \\
\hline Issue Date & 2007-10 \\
\hline Doc URL & http://hdl.handle.net/2115/30348 \\
\hline Rights & The original publication is available at www.springerlink.com \\
\hline Type & article (author version) \\
\hline File Information & A OandT S2007Rev.pdf \\
\hline
\end{tabular}

Instructions for use 


\title{
Transient occurrence of vasa-expressing cells in non-genital segments during embryonic development in the oligochaete annelid Tubifex tubifex
}

by

Atsuko Oyama • Takashi Shimizu*

\author{
A. Oyama • T. Shimizu* \\ Division of Biological Sciences, \\ Graduate School of Science, \\ Hokkaido University, \\ Sapporo 060-0810, Japan \\ *e-mail address: stak@sci.hokudai.ac.jp
}


Abstract The primordial germ cells (PGCs) in the oligochaete annelid Tubifex tubifex are mesodermal in origin and are located in the two midbody segments X and XI in which the testis and the ovary are formed, respectively. To identify a molecular marker for the Tubifex PGCs, we isolated the Tubifex homologue (Ttu-vas) of the Drosophila vasa gene. Using whole-mount in situ hybridization, we examined the spatial expression patterns of Ttu-vas from 1-cell stage through juvenile stage. Ttu-vas mRNA is present as a maternal transcript distributed broadly throughout the early stages. Ttu-vas is expressed in all of the early cleavage blastomeres, in which Ttu-vas RNA associates with mitotic spindles and pole plasms. Expression of Ttu-vas gradually becomes restricted, first to teloblasts, then to their blast cell progeny comprising the germ bands (GBs), and finally to a set of large ventral cells (termed VE cells) in a variable set of midbody segments including the genital segments (X and XI). At the end of embryogenesis, VE cells are confined to genital segments where they are presumably germline precursors in the juvenile. Staining with a cross-reacting anti-Vasa antibody suggested that VE cells express Ttu-vas protein to the same extent irrespective of their positions along the anteroposterior axis. A set of cell ablation experiments suggested that VE cells are derived from the mesodermal teloblast lineage and that the emergence of VE cells takes place independently of the presence of the ectodermal GBs that normally overlay the mesoderm. These results suggest that $T$. tubifex generates supernumerary presumptive PGCs during embryogenesis whose number is variable among embryos.

Keywords Primordial germ cells • Oligochaete annelid • Tubifex tubifex • Embryogenesis $\bullet$ vasa $\bullet$ Body segments 


\section{Introduction}

The generation of germ cells is a crucial event for all sexually reproducing animals. In most animals, it is preceded by the specification of embryonic cells as primordial germ cells (PGCs), which are defined as the first cells that exclusively give rise to germ cells by clonal mitotic divisions. The modes of PGC specification have been divided into two categories, preformation and epigenesis. While the localization of maternally inherited determinants is involved in PGC specification in the preformation mode, inductive signals from surrounding tissues have a central role in the epigenetic mode of PGC specification. These two distinct modes of PGC specification are seen in a variety of phyla. How these two modes evolved in metazoans is a challenge in evolutionary developmental biology, and still remains to be determined. On the basis of their extensive survey of existing data on PGC specification modes, Extavour and Akam (2003) have recently suggested that the epigenetic mode of PGC specification may be ancestral to the Metazoa.

Recent studies on embryonic vasa expression in a variety of animals have suggested that the developmental processes leading to PGC formation are highly diverse among animals. This diversity is manifested especially by comparing vasa expression pattern in arthropods such as fruit fly (Dme; Lasko and Ashburner 1988), red flour beetle (Tca; Schröder 2006), silk moth (Bmo; Nakano 1999), grasshopper (Sgr; Chang et al. 2002), spider mite (Tur; Dearden et al. 2003), branchiopod (Dma; Sagawa et al. 2005) and amphipod (Pha; Extavour 2005). For instance, vasa transcripts are maternally contributed to the egg in Dme, Tca and Bmo, but not in Sgr, Tur and Dma. Localization patterns of maternally supplied vasa RNA during embryonic development are also distinct among the insects: vasa RNA, which is initially distributed uniformly in the egg, either disappears in late blastoderm embryos of Dme, accumulates at the posterior pole during blastoderm formation in Tca, or is restricted to a cluster of cells located on the ventral midline of Bmo embryo. The modes of PGC formation (defined by vasa expression) in Sgr, Tur, Dma and Pha are distinct not only from those insects but also from each other. It seems that such diversity in PGC formation mode correlates to the diversity in pattern formation of embryos in these organisms. In this regard, it should be noted that medaka and zebrafish, which have almost identical body plan, exhibit different localization pattern of vasa RNA in their early embryos (Yoon et 
al. 1997; Shinomiya et al. 2000). This may suggest that strategies for PGC formation have evolved more extensively than has been thought.

The process of PGC development appears to be diverse among annelids as well. In the polychaete annelid Platynereis dumerilii, PGCs (defined by expression of vasa and nanos) arise from the mesodermal posterior growth zone at the end of larval development, and migrate into the anterior segments to form a gonial cluster (Rebscher et al. 2007). In contrast, PGCs (or presumptive PGCs defined by nanos expression) in the clitellate annelid Helobdella robusta (leech) emerge in the mesodermal germ bands during embryogenesis (Kang et al. 2002). In this leech, it has also been shown that presumptive PGCs that initially emerge in the germ band do not necessarily become PGCs, suggesting the production of 'supernumerary' presumptive PGCs. How widely this feature is shared among clitellate annelids remains to be explored. In view of the fact that the number of gonads is absolutely constant in each oligochaete family (Jamieson 2006) and that the exact number of gonads varies not only between leech species but also, albeit to lesser extent, within species (Mann 1962; Brusca and Brusca 2003), it seems likely that the two groups of clitellate annelids, i.e., oligochaetes and leeches, undergo different modes of PGC formation.

The present study was undertaken to gain an insight into the mode of PGC formation in an oligochaete annelid Tubifex tubifex. This animal has testes and ovaries in body segments X and XI, respectively (Dixon 1915). It appears that PGCs are situated in these genital segments as early as the end of embryogenesis (Meyer 1929); it has been demonstrated that these cells are derived from the mesodermal teloblast lineage (Goto et al. 1999a). The precise embryonic origin of the PGCs in T. tubifex remains to be determined, however. Based on their morphological observations on Tubifex embryos, Meyer $(1929,1931)$ indicated that PGCs arise directly from segments X and XI, while Penners and Stäblein (1930) envisaged that PGCs migrate into the segments X and XI from elsewhere (also see Shimizu 1982 for review). These earlier authors had regarded 'large cells' as PGCs. Needless to say, however, cell size alone is not sufficient as a criterion to identify PGCs. Thus, both of the previously proposed scenarios for PGC formation remain to be subjected to extensive re-examination with a reliable marker for PGCs (or germ cells).

Here, we have isolated the Tubifex homologue (Ttu-vas) of the Drosophila vasa gene, and, using whole-mount in situ hybridization technique, examined the spatial 
expression patterns of Ttu-vas from 1-cell stage through to juvenile stage. We find that maternally contributed Ttu-vas RNA associates with mitotic spindles and pole plasms in the early cleavage blastomeres. This RNA is segregated, via teloblasts, to blast cells, and finally restricted to ventrally located large cells. We also find that the number and the position (along the anteroposterior axis) of such ventral Ttu-vas-expressing cells are highly variable among embryos and that nearly all of the cells but those in segments $\mathrm{X}$ and XI disappear by the end of embryogenesis. We show that ventral Ttu-vas-expressing cells are derived from the mesodermal teloblast lineage and that not only the appearance of but also the ensuing disappearance of these cells occur independently of the overlying ectoderm.

\section{Materials and methods}

Embryos

Embryos of the freshwater oligochaete Tubifex tubifex were obtained as described previously (Shimizu 1982) and cultured at $22^{\circ} \mathrm{C}$. For experiments, embryos were freed from cocoons in the culture medium (Shimizu 1982). Sterilization of cocoons (embryos) and blastomere ablation were done according to Nakamoto et al. (2004). Unless otherwise stated, all experiments were carried out at room temperature $\left(20-22^{\circ} \mathrm{C}\right)$.

Inhibitors

An aqueous stock solution of actinomycin D (Calbiochem) was prepared at a concentration of $2 \mathrm{mg} / \mathrm{ml}$, and aliquots were stored at $-20^{\circ} \mathrm{C}$. A stock solution of nocodazole (Janssen) was prepared with DMSO at a concentration of $10 \mathrm{mg} / \mathrm{ml}$, and stored at $-20^{\circ} \mathrm{C}$. Immediately before use, small volumes of these stock solutions were diluted with the culture medium.

Degenerate PCR and 3' Rapid amplification of cDNA ends (RACE)

RNA isolation and purification and cDNA synthesis were performed according to 
Matsuo et al. (2005). To clone the vasa gene of T. tubifex, we designed degenerate PCR primers based on the amino acid sequences conserved among the vasa-class genes of invertebrates (Drosophila melanogaster and Ciona intestinalis) and vertebrates (Gallus gallus, Danio rerio and Homo sapiens). Forward primers, F1 and F2, corresponded to amino acid sequences GINFDKYD and MACAQTG, respectively; reverse primers, R1 and R2, corresponded to QTLMFSAT and MLDMGF, respectively. Nucleotide sequences of these primers were 5'-GGNATHAAYTTYGAYAARTAYG-3' (F1), 5'-ATGGCITGYGCNCARACNGG-3' (F2),

5'-GTNGCRCTRAACATIARNGTYTG-3' (R1), and

5'-AANCCCATTRTCNARCAT-3' (R2). Detailed protocols and amplification parameters for the degenerate PCR are available upon request.

To isolate the 3' portion of vasa transcripts, 3' RACE was performed using gene specific primers (GSPs) and an adapter primer (5’-AACTGGAAGAATTCGCGGCC-3'). GSPs for vasa are designated Ttu-vasa GSP1 (5’-TTCCTGCTTCCGGTTCTGAC-3') and Ttu-vasa GSP2 (5'-GGAATGCTACGCAACGG-3'). Detailed protocols and amplification parameters for the 3' RACE are available upon request.

Relative quantification of gene expression by RT-PCR

The mRNA was extracted from various developmental stages and reverse transcribed into single-strand cDNA (First-Strand cDNA Synthesis Kit; Amersham Biosciences), which was used as a template for RT-PCR. PCR was performed on cDNA with primers Ttu-vasa GSP3 (5’-ACCTCGGTCGTGCTACCCA-3') and Ttu-vasa GSP4 (5'-GGACCAAGAAGAGTCAAATG-3'). Amplification parameters were: $2 \mathrm{~min}$ at $95^{\circ} \mathrm{C}, 30$ cycles of $30 \mathrm{~s}$ at $95^{\circ} \mathrm{C}, 30 \mathrm{~s}$ at $58^{\circ} \mathrm{C}$ and $60 \mathrm{~s}$ at $72^{\circ} \mathrm{C}$, and final extension at $72^{\circ} \mathrm{C}$. $\quad \beta$-actin was used as positive control (Matsuo and Shimizu, 2006).

Amplification parameters for $\beta$-actin were the same as those for vasa, except that the number of cycles was 25 . Reactions were agarose-gel electrophoresed, stained with ethidium bromide, and photographed under UV light.

RNA probe synthesis and whole-mount in situ hybridization 
Digoxigenin (DIG)-labeled RNA probes were prepared according to the method described by Matsuo et al. (2005).

For whole-mount in situ hybridization, embryos and juveniles were processed according to the protocols described by Matsuo et al. (2005), except that hybridization was performed with $0.5-1 \mu \mathrm{g} / \mathrm{ml}$ RNA probe at $60^{\circ} \mathrm{C}$ for $48 \mathrm{~h}$. Whole-mount processed (stained) embryos were mounted in PBST (PBS+0.1\% Tween-20) and observed under incident light. Some stained embryos were cleared according to the method described by Matsuo and Shimizu (2006) and observed with transmitted light.

Whole-mount immunocytochemistry

Immunocytochemical whole-mounts of fixed embryos (stages 1-17) were prepared according to Shimizu (1993) and Shimizu and Savage (2002), with some modifications. Embryos at stage 18 and juveniles (after fixation) were treated sequentially with 5\% mercaptoethanol and $1000 \mathrm{U} / \mathrm{ml}$ collagenase (Type VII; Sigma ) according to Yoshida-Noro et al. (2000). A rabbit polyclonal anti-Vasa antibody (kindly donated by Drs. C. G Extavour and M. Akam) raised against Schistocerca (grasshopper) Vasa protein was used as a primary antibody; a goat anti-rabbit IgG antibody conjugated to horseradish peroxidase (HRP; Sigma) was used as a secondary antibody.

\section{Results}

Summary of Tubifex development

A brief review of Tubifex development is presented here as a background for the observations described below (for details, see Shimizu 1982; Goto et al. 1999a, b). Tubifex eggs, which are oviposited at metaphase of the first meiosis, undergo polar body formation twice and then enter the first mitosis. Before the first cleavage, yolk-deficient cytoplasm called pole plasm accumulates at both poles of the egg (Fig. 1a). The early development of Tubifex consists of a stereotyped sequence of cell divisions (Shimizu 1982). The first cleavage of the Tubifex egg is unequal and meridional, and produces a smaller AB-cell and a larger CD-cell (Fig. 1b). The second cleavage is also meridional and yields cells A, B, C and D: the CD-cell divides into a 
smaller C-cell and a larger D-cell while the AB-cell separates into cells A and B of various sizes (Fig. 1c). From the third cleavage on, the quadrants A, B and C repeat unequal divisions three times, and the $\mathrm{D}$ quadrant four times, producing micromeres at the animal side and macromeres at the vegetal side (Fig. 1d, e). The quadrants A, B and $\mathrm{C}$ then divide equally at the sixth cleavage, followed by the D quadrant at the seventh cleavage; the resulting yolky macromeres are endodermal cells, and these repeat equal divisions thereafter. During cleavages, the pole plasms are inherited by the D lineage cells; they are finally partitioned into the second (2d) and fourth (4d) micromeres. At the 24-cell stage, $2 \mathrm{~d}^{11}, 4 \mathrm{~d}$ and $4 \mathrm{D}$ (sister cell of $4 \mathrm{~d}$ ) all come to lie in the future midline of the embryo (Fig. 1f). $4 \mathrm{~d}$ divides equally to yield the left and right mesoteloblasts ( $\mathrm{Ml}$ and $\mathrm{Mr}$ ); 2d $\mathrm{d}^{111}$ (derived from $2 \mathrm{~d}^{11}$ ) divides into a bilateral pair of ectoteloblast precursors, NOPQl and NOPQr; and 4D divides equally yielding endodermal precursors $\mathrm{E}^{\mathrm{D}}$ (Fig. 1g). Ectoteloblasts N, O, P and Q arise from an invariable sequence of divisions of cell NOPQ on both sides of the embryo (Fig. 1h; for details see Nakamoto et al. 2004): the $\mathrm{N}$ teloblast is generated first and located ventralmost (stage 12a), and the Q teloblast, which is generated next, is located dorsalmost (stage 12b); finally the $\mathrm{O}$ and $\mathrm{P}$ teloblasts are generated by almost equal division of their precursor cell, at which point teloblastogenesis is complete (stage 12c).

After their birth, each of the teloblasts thus produced divides repeatedly, at 2.5-hour intervals (at $22^{\circ} \mathrm{C}$ ), to give rise to small cells called primary blast cells, which are arranged into a coherent column (i.e., a bandlet). Within each bandlet, primary blast cells and their descendants are arranged in the order of their birth. Bandlets from $\mathrm{N}, \mathrm{O}, \mathrm{P}$ and Q teloblasts on each side of the embryo join together to form an ectodermal $\mathrm{GB}$, while the bandlet from the M teloblast becomes a mesodermal GB that underlies the ectodermal GB. The GBs are initially located on the dorsal side of the embryo (Fig. 1i). Along with their elongation, they gradually curve round toward the ventral midline (Fig. 1j) and finally coalesce with each other along the ventral midline (Fig. 1k). The coalescence is soon followed by dorsalward expansion of the GBs. The edges of the expanding GBs on both sides of the embryo finally meet along the dorsal midline to enclose the yolky endodermal tube (Fig. 1l-q). Concurrently with this enclosure, the embryo becomes elongated in an anterior-to-posterior progression, and curved with the ventral convexity (Fig. 1l-q). Enclosed portions of the embryo begin to exhibit peristaltic movements. Embryogenesis is judged to complete when the expanding GBs 
have enclosed the posterior end of the embryo, which then exhibits movement throughout its length (Fig. 1q).

Isolation and characterization of a vasa homologue from Tubifex tubifex

Using degenerate PCR, we amplified fragments (384 bp) of a vasa gene from Tubifex tubifex, and used 3'RACE to obtain additional sequence of this gene, designated Ttu-vas (accession number AB205013). The domain of Ttu-vas flanked by motifs that are conserved in vasa-class genes of many animals, shows 50-66\% amino acid identity with those of other vasa-class genes (Fig. S1a). Ttu-vas contains EARKF and WD motifs characteristic of vasa-class genes, in addition to the other (eight) motifs conserved in RNA helicase genes (Fig. S1a). An analysis of the phylogenetic relationships of the predicted Ttu-vas animo acid sequence indicated that it clusters with Vasa, as opposed to other RNA helicase proteins, from both protostomes and deuterostomes (Fig. S1b; bootstrap support 94\%). Ttu-vas is thus clearly a true vasa homologue.

Temporal expression pattern of Ttu-vas

The temporal expression profile of Ttu-vas was analyzed by RT-PCR. The results are shown in Fig. 2. Ttu-vas mRNA was present at detectable and unvaried levels from 1-cell stage through stage 12. Thereafter Ttu-vas transcripts decreased in amount to undetectable levels at stage 17, when embryos undergo the second episode of body elongation (see Fig. 1q). It should be noted that Ttu-vas-expressing cells are detectable in late stage embryos (stages 16 to 18) and even in juveniles albeit few in number in individual embryos (see below).

Spatial expression patterns of Ttu-vas in early cleavage blastomeres

In many animals, it has been shown that vasa-related genes are specifically expressed in germline cells (Extavour and Akam, 2003). To find out if this is the case for Tubifex tubifex, we examined the spatial expression patterns of Ttu-vas during Tubifex embryogenesis, using whole-mount in situ hybridization techniques. Whole-mount 
staining with antisense and sense riboprobes revealed the presence of easily detectable level of Ttu-vas RNA throughout the embryonic stages up to early gastrula. During early stages, Ttu-vas transcripts were present mainly in the pole plasm domains and mitotic spindles. The pole plasm-associated Ttu-vas RNA was inherited by blastomeres of the D-cell line: the animal and vegetal pole plasm domains in the 1-cell stage (Fig. 3a-c); CD cell in the 2-cell stage (Fig. 3e); D cell in the 4-cell stage (Fig. $3 \mathrm{~h}) ; 2 \mathrm{~d}, 2 \mathrm{D}$ and $3 \mathrm{D}$ in the 12-cell stage (not shown); 2d and 4d in the 24-cell stage (Fig. 3i).

The association of Ttu-vas RNA with the mitotic spindle was seen in nearly all of the blastomeres of the C- and D-cell lines up to stage 11 (Fig. 3d, e, f, h-m). It is of interest to note that Ttu-vas transcripts were more concentrated to the spindle poles (i.e., the centrosomal regions) than any other regions of the mitotic apparatus (Fig. 3f, k, 1). Ttu-vas RNA in the AB cell and its descendants also appeared to associate with mitotic spindle, but the signal was often too weak to outline the spindle (see cells A and $\mathrm{B}$ in Fig. 3h).

In summary, we suggest that Ttu-vas RNA in early blastomeres is concentrated in the mitotic spindle and pole plasms. Given that microtubules are major components of the mitotic spindle, this mRNA concentration to the spindle could be mediated by microtubules. To test this possibility, we examined the Ttu-vas RNA distribution in 2-cell embryos that had been treated with $5 \mu \mathrm{g} / \mathrm{ml}$ nocodazole for $1 \mathrm{~h}$. Fig. $4 \mathrm{~g}$ shows a representative of such treated embryos. Ttu-vas RNA in CD cell of treated embryos was found to accumulate at pole plasms and cell cortex but not elsewhere. Apparently the concentration of Ttu-vas RNA seen in the spindle of control embryos (see Fig. 3e) was lacking in the treated embryos.

Spatial expression pattern of Ttu-vas RNA during teloblastogenesis

During teloblastogenesis, Ttu-vas RNA was detected in proteloblasts, teloblasts, and blast cells generated therefrom. It was also found in some micromeres that were located around a “cap”, comprised of cells $1 \mathrm{a}, 1 \mathrm{~b}, 1 \mathrm{c}, 1 \mathrm{~d}$ and $2 \mathrm{~d}^{112}$; the level of mRNA in cells of the micromere cap was relatively very low so that this cap appeared as a Ttu-vas-negative circular domain (Fig. 4a-d). Macromeres exhibited Ttu-vas expression to some extent during early phase of teloblastogenesis (Fig. 3m); however, 
Ttu-vas RNA in macromeres was undetectable as early as the time of division of NOPQ into $\mathrm{N}$ and OPQ (Fig. 4e).

In proteloblasts and teloblasts, Ttu-vas RNA was located at their anterior regions (Fig. 3q). As in earlier blastomeres, when these cells entered mitosis, Ttu-vas RNA was concentrated in the mitotic spindle (Fig. 4c, f, h). During teloblastogenesis (stages 11 and 12), not only teloblasts are generated, but primary blast cells (and their progeny) produced from teloblasts and proteloblasts also form incipient ectodermal and mesodermal GBs. As Fig. 4e-h shows, these early GBs exhibit rather strong signals of Ttu-vas staining. The level of Ttu-vas RNA in primary blast cells was comparable to that in teloblasts (Fig. 4d, e, f, h). Ttu-vas RNA in interphase blast cells appeared to be distributed evenly throughout the cytoplasm (Fig. 4d); however, we could not determine whether Ttu-vas transcripts are concentrated to the spindle in dividing primary blast cells.

Occurrence of ventral Ttu-vas-expressing cells during gastrulation

After teloblastogenesis completes, Tubifex embryos undergo gastrulation (stages 13 to 15), during which GBs elongate, curve round toward the ventral midline and coalesce along it. Ttu-vas expression in embryos at these stages was detected in GBs exclusively. It is apparent, however, that staining intensity of GBs became lower as development proceeded during stages 13 to 15 (Fig. 5a-d). As Fig. 5e, f shows, embryos at the end of stage 15 exhibited only a trace of Ttu-vas expression in GBs (except ventral large cells; see below). It should be noted that the comparisons of the in situ staining were made among embryos (at different stages) that had been processed for the in situ hybridization and carried through the color reaction in the same tube.

The most prominent change occurring in the GBs was the emergence of several large cells with relatively strong signal in the mesodermal GBs (Fig. 5d). These Ttu-vas-expressing cells were first recognizable (in whole-mount preparations) only when anterior halves of GBs completed coalescence along the ventral midline. These cells were present approximately at the midpoint along the ventral midline (Fig. $5 d$ ); in whole-mount preparations (whether they were cleared or not), however, it was difficult to determine precisely the positions of these cells with respect to those of segments in stage 15 embryos (Fig. 5c, d). Judging from the fact that the segments VII 
and VIII are located in the midzone of the anterior half of the late stage 15 Tubifex embryos (Kitamura and Shimizu 2000a), it is safe to say that Ttu-vas-expressing cells in stage 15 embryos were located posterior to the segment VIII. Thus we tentatively suggest that such ventral Ttu-vas-expressing cells emerge in the region corresponding to segments IX to XII. The ventrally localized Ttu-vas-expressing large cells will be hereafter referred to as "VE cells".

The emergence of the VE cells could result from specific upregulation of Ttu-vas transcription in such cells. Alternatively the emergence of VE cells could result indirectly from downregulation of Ttu-vas in cells other than the VE cells. To differentiate these possibilities, we treated embryos at the beginning of stage 13 with $200 \mu \mathrm{g} / \mathrm{ml}$ actinomycin D, fixed them after $48 \mathrm{~h}$ treatment, and examined them for VE cells. A previous study showed that actinomycin D (at a concentration of $50 \mu \mathrm{g} / \mathrm{ml}$ ) treatment is sufficient to abrogate zygotic expression of Tubifex homologue of dorsal (Matsuo et al. 2005). Control embryos were immersed in 0.9x culture medium for 48 $\mathrm{h}$ before fixation. Development of control embryos was completely normal at the time of their fixation and it reached stage 16a (see Fig. 5e, g).

Fig. 5i-m show representative embryos treated with actinomycin D. Judging from their morphology, it is apparent that actinomycin D treatments introduced in this study impaired the elongation of and the accompanying morphogenetic movements of GBs. It seemed that development of the treated embryos was halted at stage 13 . In spite of such impairement, all of the treated embryos $(n=30)$ exhibited Ttu-vas-expressing large cells which were indistinguishable from VE cells in intact (control) embryos (compare Fig. 5j-m with Fig. 5g, h). These results suggest that de novo transcription of Ttu-vas is not required for the emergence of VE cells.

Behavior of ventral Ttu-vas-expressing (VE) cells during embryo’s body elongation

Ttu-vas-expressing large cells (VE cells) that were very similar to those seen in stage 15 embryos were found on the ventral side of embryos at stage 16 through to juvenile stage. Individual VE cells were located in the interior regions (but not on the surface) of the embryo, and they were visible more clearly from outside than before in whole-mount preparations. This may be partly because cell layers overlying VE cells become thinner than before as a result of progress of cell division in ectodermal and mesodermal 
GBs (see Goto et al. 1999b; Nakamoto et al. 2000). Since embryos at stage 16 or later exhibit morphological periodicity along the ventral margin corresponding to the boundary between adjacent segments (which are defined here according to mesodermal somites), it was possible to locate VE cells with respect to the position of segments under a dissecting microscope. VE cells were individually located in the anterior half of a segment. It should be noted that although single VE cells were seen in individual hemisegments in most cases, there were found some cases where two VE cells were present in a single hemisegment. The staining intensity of VE cells showed little variation within an embryo, between embryos at the same developmental stage, or even between different stages.

Distribution patterns of VE cells along the anteroposterior axis. At stage 16, VE cells were confined to the regions ranging from segment IX to XII (Fig. 6).

Although VE cells were always located in two, three or four consecutive hemisegments on either side of the embryo, the position of the anteriormost and posteriormost hemisegments containing VE cells varied from embryo to embryo and even from side to side within individual embryos. Considering the positions of these cells along the anteroposterior axis (i.e., with respect to the positions of segments) on either side of the embryo, 12 patterns of their distribution were discernible (Fig. 6). Fig. 8a shows the frequency of VE cells in each of the segments V to XII on both the left and right sides of the embryo. It is evident that all of the embryos examined exhibited VE cells in segments X and XI. In contrast, the occurrence of VE cells in either segment IX or XII occurred only in 16 to $30 \%$ of embryos examined. There were some embryos in which all of the four consecutive segments (IX-XII) on both sides exhibited VE cells (Fig. 6a).

During subsequent development Tubifex embryos underwent three episodes of changes in the occurrence of VE cells. The first episode is the increase in the frequency of VE cells in segments IX and XII, which occurred during stages 16b and 16c (Fig. 7b, c). The second episode is the additional occurrence of VE cells in the regions ranging from segment V to VIII, which took place during stages 16c and 17a (Fig. 7c, d). It should be noted that only about $20 \%$ of embryos examined exhibited VE cells in the regions anterior to segment IX (10/56 cases at stage 16c; $13 / 62$ cases at stage 17a). The third episode, which lasted for a rather long time beginning at stage 17b through early stage of juvenile, was that VE cells became undetectable in segments 
other than X and XI (Fig. 7e-k). Consequently, in more than $90 \%$ of juveniles examined, VE cells were seen exclusively in segments X and XI but not in any other segment (Fig. 7g, 8a, b). In the remaining juveniles, Ttu-vas-positive tiny dots were observed in one or two segments other than segments X and XI (Fig. 8c-e). In this study we regarded these dots as VE cells and included them in Fig. 7g-k (as indicated by asterisks). However, it should be noted that these dots were much smaller than "authentic" VE cells (see Fig. 8c-e).

Number of VE cells on one side of an embryo. During embryogenesis (i.e., stages 16 and 17), the occurrence of three VE cells was more frequent than any other value of the VE cell number on both the left and right side (Fig. S2). Following the completion of embryogenesis, nearly all of the juveniles exhibited two VE cells on either side; as described before, these two cells were localized in segments X and XI (Fig. S2).

A cross-reacting Vasa antibody stains VE cells

The aforementioned observations suggest that as to the mode of Ttu-vas expression, there are two classes of VE cells. One class consists of genital segment-associated VE cells in which Ttu-vas expression persists through to juvenile stage; the other comprises non-genital segment-associated VE cells from which Ttu-vas transcripts disappear by the end of embryogenesis. As described before, there were no significant differences in staining intensity for Ttu-vas transcripts among VE cells.

We were interested in learning whether these two classes of VE cells differ from each other in translational level of this gene. In this study, to gain an insight into expression of Ttu-vas protein, in terms of the Ttu-vas transcripts, we stained Tubifex embryos with an anti-Vasa antibody named formosa 2, which was raised to Schistocera Vasa protein (Chang et al. 2002). According to these authors, the amino acid sequence of the polypeptide used to raise this antibody included six of the eight conserved DEAD-box protein motifs as well as the EARKF motif; we find that this sequence shows 53\% identity to the corresponding domain of the predicted Ttu-vas protein (data not shown). In a preliminary experiment, we found that formosa 2 antibody specifically stains PGCs in segments X and XI of Tubifex juveniles (Fig. 9h). We suggest that this antibody recognizes antigen related to Ttu-vas protein. 
Ttu-vas protein was detected in all blastomeres from stage 1 through to stage 13 (Fig. 9a-c). It was found to be concentrated in the perinuclear region in every blastomere; in blastomeres that inherit pole plasms, it was also concentrated in the pole plasm domain (Fig. 9a). As development proceeded during stages 14 to 16, the perinuclear localization of Ttu-vas protein declined in an anterior-to-posterior progression, leaving some ventrally located large cells with strong signal for Ttu-vas protein.

As Fig. 9d-g shows, ventrally located large cells that were formosa 2-positive were distributed approximately in the mid region of embryos at stages 16 and 17. Taking their size and location in each segment into consideration, it is likely that these cells correspond to the aforementioned Ttu-vas-expressing (VE) cells. Furthermore, the distribution pattern of stained cells along the anteroposterior axis appeared to be comparable to that seen in VE cells. For instance, stained cells were seen in segments $\mathrm{X}$ and XI in all of the embryos examined; about 70\% of embryos examined exhibited stained cells in segment XII but the remaining embryos did not. In any of the embryos examined, there were no significant differences in staining intensity among formosa 2-positive cells (Fig. 9d-g). This suggests it is unlikely that the two classes of VE cells differ from each other at the translational level.

Ventral Ttu-vas-expressing cells may originate in the mesodermal germ band

As described before, VE cells are located deep inside but not on the embryo's surface. This location corresponds to the mesodermal GB. Given that the mesodermal GB in the Tubifex embryo is originated exclusively from mesoteloblasts M (Goto et al. 1999b), it is possible that VE cells are mesodermal in origin and derived from $\mathrm{M}$ teloblasts. To test this possibility, we performed cell ablation experiments in which embryos were deprived of precursors (cells $2 \mathrm{~d}^{111}$ and $4 \mathrm{~d}$ ) of teloblasts, cultured for 5 or 10 days, and examined for Ttu-vas-expressing cells. Control embryos were allowed to develop without vitelline membrane, and reached stages 17b and Juv-D3 at 5 and 10 days of operation, respectively. As Fig. 10a, b shows, VE cells developed in a normal fashion in control embryos.

The ablation of $4 \mathrm{~d}$ cells resulted in the absence of $\mathrm{M}$ teloblasts, hence, mesodermal GBs. These operated embryos underwent cell divisions and ectodermal 
GB formation in a normal fashion, though they failed to elongate (Kitamura and Shimizu 2000b). As Fig. 10c, d shows, there was no trace of Ttu-vas-positive cells in these embryos either at 5 days (20/20 cases) or 10 days (15/15 cases) after cell ablation.

In contrast, embryos that had been deprived of $2 \mathrm{~d}^{111}$ cells (precursor of ectoteloblasts N, O, P and Q) underwent body elongation to some extent (Fig. 10e, f). At 5 days after cell ablation, distinct Ttu-vas-expressing cells (2 to 4 in number on either side) were seen in all of the operated embryos (12/12 cases), though it was difficult to determine their location with respect to the positions of segments (Fig. 10e). At 10 days, two pairs of VE cells were found in operated embryos (10/10 cases; Fig. 10f).

These results suggest that ventrally located Ttu-vas-expressing cells, i.e. VE cells, in the Tubifex embryo may originate in the mesodermal GB but not in the ectodermal GB. Furthermore, given that VE cells emerge in the absence of the ectodermal GB (Fig. 10e, f), it seems unlikely that VE cells develop in the mesodermal GB via some kind of induction from the ectodermal GB. At present, however, we cannot eliminate another possibility that a subset of VE cells arise from ectodermal GBs under the influence of mesoderm.

\section{Discussion}

Subcellular localization of Ttu-vas mRNA in early blastomeres

The present study shows that Ttu-vas RNA in early blastomeres associates with the mitotic spindle and pole plasms. Although Ttu-vas RNA is distributed along the length of the spindle, it is more concentrated at the spindle poles than any other regions of the spindle. Given that centrosomes are present at both poles of the spindle (Shimizu 1996), it is conceivable that Ttu-vas RNA is localized to the centrosomal regions. Similar localization of mRNAs to the centrosomes in embryonic cells has been reported for some developmental patterning genes (dpp and eve) in the mollusc Ilyanassa obsoleta (Lambert and Nagy 2002). Unlike Ttu-vas RNA, however, these mRNAs do not appear to associate with the mitotic spindle itself.

As suggested from the experiments with the microtubule inhibitor nocodazole (Fig. 3g), the localization of Ttu-vas RNA to the mitotic spindle is mediated by 
microtubules. In the same nocodazole-treated embryos, however, the distribution of Ttu-vas RNA associated with the pole plasms did not appear to be affected. This may suggest the involvement of cytoskeleton other than microtubules in the integration of Ttu-vas RNA in the pole plasm. We suspect that actin networks serve this cytoskeletal function, since pole plasms contain an elaborated actin network (Shimizu, 1995).

Embryonic expression of vasa-related genes has been studied in a variety of animals, and it has been shown that the modes of maternally supplied vasa RNA distribution in early blastomeres and embryos vary among animals. In some organisms such as sea anemone, polychaete and medaka, maternally supplied vasa RNA is inherited by all of the early blastomeres and distributed evenly in each blastomere (Shinomiya et al. 2000; Extavour et al. 2005; Rebscher et al. 2007). By contrast, at very early stages of development in red flour beetle, ascidians and some other teleosts (such as zebrafish, goldfish and loach), vasa RNA becomes localized into certain subcellular structures in early blastomeres (Yoon et al. 1997; Braat et al. 2000; Fujimura and Takamura 2000; Knaut et al. 2000; Krфvel and Olsen 2002; Otani et al. 2002; Fujimoto et al. 2006; Schröder 2006; Shirae-Surabayashi et al. 2006). In the oyster Crassostrea gigas, vasa RNA accumulates at the vegetal region of the egg before the first cleavage; during the ensuing cleavages, it is associated with perinuclear region, inherited by the D quadrant, and finally segregated to the $4 \mathrm{~d}$ mesentoblast (Fabioux et al. 2004).

Although, as summarized above, a variety of subcellular localization patterns have been reported for vasa RNA, such association of vasa RNA with a mitotic spindle as seen in Tubifex has not been mentioned in any of the organisms studied so far. This may suggest that the localization (or concentration) of vasa RNA to the spindle is unique to Tubifex. On the other hand, the involvement of intact microtubules in mRNA localization appears to be widespread, since this role for microtubules has been demonstrated in the translocation of vasa-RNA-containing aggregates in zebrafish (Pelegri et al. 1999), in the localization of dpp mRNA to the centrosome in Ilyanassa obsoleta embryo (Lambert and Nagy 2002), in the localization of bicoid RNA to the anterior pole and oskar RNA to the posterior pole of the Drosophila egg (Pokrywka 1995), and in the translocation of transcripts of $\mathrm{Vg} 1$ and Xcat-2 to the vegetal cortex of the Xenopus oocyte (Yisraeli et al., 1990; Zhou and King, 1996). As suggested from reintroduction experiments with in vitro synthesized RNA, the 3’UTR of bicoid and 
Xcat-2 RNAs contains information both required and sufficient for localization (Pokrywka 1995; Zhou and King 1996). Therefore, it would be of interest to examine whether the 3'UTR of the Ttu-vas RNA is involved in Ttu-vas RNA localization to the mitotic spindle.

Inheritance of maternally supplied Ttu-vas RNA by blast cells

Although the present RT-PCR analysis was not strictly quantitative, the results obtained suggest that the level of Ttu-vas RNA present in individual embryos does not change significantly during early development (stages 1 to 13 ). It is unlikely that this maintenance of the Ttu-vas RNA level depends on de novo transcription of this gene, because embryos that had been treated with $200 \mu \mathrm{g} / \mathrm{ml}$ actinomycin $\mathrm{D}$ for 6 hrs either at stage 1, 5, 8 or 11 exhibited localization pattern of and staining intensity for Ttu-vas RNA, both of which were comparable to those in intact embryos (as shown in Fig. 4, 5) irrespective of time points of treatment (unpublished observation). We thus envisage that a significant portion of maternally supplied Ttu-vas RNA is inherited by early blastomeres of the D cell line, segregated to cells $2 \mathrm{~d}$ and $4 \mathrm{~d}$, and finally partitioned to blast cells that will form GBs.

Emergence of ventral Ttu-vas-expressing (VE) cells

One of the most prominent events occurring during gastrulation is the appearance of ventrally located Ttu-vas-expressing (VE) cells. As suggested from the experiments with actinomycin $\mathrm{D}$, it is unlikely that the VE cell emergence depends on de novo transcription of Ttu-vas. It is conceivable that when VE cells emerge, the level of Ttu-vas RNA they contain remains unchanged. Nevertheless, VE cells become "visible" in the GBs. This may be simply because Ttu-vas expression declines in cells comprising GBs except for those to be VE cells. In fact, staining of GBs in whole-mount preparations appears to be weaker as development proceeds during stages 13 to 16. RT-PCR analysis also showed that the bulk of Ttu-vas RNA disappears from embryos by the end of stage15 (Fig. 2). Given that maternally contributed Ttu-vas RNA is segregated, via teloblasts, to blast cells that form GBs, it is highly possible that Ttu-vas RNA persisting in such initial VE cells as seen at stage 15 is maternal in origin. 
Thus, as to the fate of maternally supplied Ttu-vas transcripts, we suggest that if they are segregated to cells that are to be VE cells they persist therein, and that otherwise, they are subjected to degradation during early stages of gastrulation.

Similar differential degradation of transcripts among embryonic cells has also been suggested for vasa genes in the medaka (Shinomiya et al. 2000), the silkmoth (Nakano 1999) and the polychaete (Rebscher et al. 2007) and even for nanos gene in the leech (Kang et al. 2002).

Mechanisms for this differential degradation remain to be explored. In the zebrafish, it has been demonstrated that vasa mRNA, which is rapidly degraded in somatic cells, is stabilized in the PGCs in a process that is mediated by cis-acting elements within the molecule (Wolke et al. 2002). Similar posttranscriptional degradation-protection mechanisms could operate in Tubifex embryos as well, although, as discussed later, all of the VE cells that initially appear in the GB do not necessarily develop into PGCs.

"Stochastic" occurrence of VE cells in non-genital segments

From stage 15 onward, Ttu-vas expression is confined to ventrally localized large cells, i.e., VE cells. The present observation revealed that the number of and the distribution pattern of the VE cells along the anteroposterior axis vary considerably among embryos at any time point of development. What is shared among embryos is just that the segments $X$ and XI both exhibit VE cells without any exception. This may suggest that VE cells in non-genital segments occur stochastically while the occurrence of VE cells in genital segments (X and XI) is deterministic. Furthermore, the occurrence of VE cells in non-genital segments might be controlled differently in different regions, since the frequency of VE cells in segments IX and XII is much higher than that in segments V to VIII ( 60\% versus 25\%; see Fig. 7). Since it is not until stage 16c that VE cells become detectable in segments V to VIII (Fig. 7c), it is natural to predict that this appearance of VE cells depends on zygotic expression of Ttu-vas (though we did not address this issue in the present study). As to VE cells in segments IX and XII, it is not known whether their appearance depends on zygotic expression of Ttu-vas, although a subset of these cells are expected to inherit maternal Ttu-vas RNA upon their “emergence”. 
Another interesting observation from this study is that upon completion of embryogenesis, nearly all of the VE cells (as defined by Ttu-vas expression) disappear rather swiftly from the non-genital segments. It is apparent that these cells either die around the time of completion of embryogenesis, or cease Ttu-vas expression but exist even after embryogenesis. In this regard, Ttu-vas-positive tiny dots that are seen in early juveniles (Fig. 8c-e) deserve to be mentioned, because they look like remnants of VE cells. Assuming that such tiny dots represent apoptotic cells, it seems likely that at least a subset of VE cells located in non-genital segments are fated to die at the end of embryogenesis.

Embryonic origin of PGCs

In Tubifex tubifex segments X and XI are genital segments, in which the testis and the ovary are formed, respectively (Dixon 1915; Shimizu 1982). A previous cell-lineage study has shown that PGCs, which are located in segments X and XI of stage 18 embryos, are derived from the mesodermal teloblast (M) lineage (Goto et al. 1999a). The present study suggests that the VE cells that locate in segments X and XI around the completion of embryogenesis are specified as PGCs. In other words, PGCs in T. tubifex are the cells which uniquely maintain Ttu-vas expression after completion of embryogenesis.

VE cells detectable during embryogenesis are distinct from other cells with respect to Ttu-vas expression, but they are similar to each other in their morphology as well as the levels of Ttu-vas expression. Given that two pairs of cells that are to be specified as PGCs are selected from a population of Ttu-vas-expressing cells (i.e., VE cells), it is safe to say that VE cells are regarded as presumptive PGCs (pre-PGCs).

Embryonic origin of Tubifex PGCs (i.e., VE cells fated to be PGCs) remains to be explored, however. VE cells born in segments X and XI could be specified in situ as PGCs. Alternatively, VE cells that have migrated to these two segments from elsewhere could become PGCs therein. Our previous cell-lineage analysis (on stage 14-15 embryos) showed that each mesodermal segment is composed of descendants of a single primary m-blast cell and that there is no intermingling of cells between adjacent segments (Goto et al. 1999b). On the basis of these observations, it is tempting to favor the former possibility. At present, however, the latter possibility still remains 
feasible, since it is not known whether VE cells migrate along the GB during later stages. Thus, to differentiate these two possibilities, further studies, especially aimed at elucidating the dynamics or behavior of VE cells in individual embryos, are required.

Comparisons with other clitellate annelids: 'supernumerary' presumptive PGCs

If, as discussed above, VE cells seen during Tubifex embryogenesis are pre-PGCs, it is evident that T. tubifex generates 'supernumerary' pre-PGCs during embryogenesis although the number of such cells varies among embryos. While how widely this feature is shared by oligochaetes remains to be explored, a recent study by Kang et al. (2002) has suggested that similar 'supernumerary' pre-PGCs occur in the leech Helobdella robusta. These authors showed that 11 paired sets of pre-PGCs (defined by nanos expression) appear during embryogenesis of this leech and that only a subset of these pre-PGCs (4 to 6 paired sets) participate in gonadogenesis to form testisacs. This suggests that 'supernumerary' pre-PGCs that are not to be specified as PGCs are formed in embryos of $H$. robusta as well. At present, it is unclear whether T. tubifex and $H$. robusta serve as representatives of oligochaetes and leeches, respectively. However, the occurrence of 'supernumerary' pre-PGCs in both the oligochaete and the leech may suggest that the formation of 'supernumerary' pre-PGCs during embryogenesis is an ancestral feature among clitellate annelids.

As to the dynamics of pre-PGCs, however, there are significant differences between Tubifex and Helobdella. In Tubifex, two paired sets of pre-PGCs are invariably recruited to the germline though the number of pre-PGCs (defined by vasa expression) that are formed during embryogenesis is not fixed, but it is highly variable among embryos. In Helobdella, conversely, a fixed number (11 paired sets) of pre-PGCs (defined by nanos expression) are formed; the number of pre-PGCs that are recruited to the germline is not fixed but varies among embryos (Kang et al., 2002). These differences may be ascribable to the different marker genes used. Alternatively, these differences may be reflections of changes that might have occurred during the evolutionary isolation of oligochaetes and leeches. Most of the extant oligochaetes (specifically, in 20 out of 21 oligochaete families) exhibit 2 to 4 pairs of gonads though the remaining family Lutodrilidae has exceptionally 11 pairs of gonads (Jamieson, 2006); the number of gonads is absolutely constant in each family. In contrast, the 
extant leeches usually exhibit 5 to 10 pairs of testes. Furthermore, the exact number of testisacs varies not only between species but also, albeit to lesser extent, within species (Mann, 1962; Brusca and Brusca, 2003; Kang et al., 2002). Presumably, during their evolutionary isolation, oligochaetes and leeches have preserved an ancestral mode of pre-PGC formation despite the divergence of modes of recruitment of pre-PGCs to the germline.

Acknowledgements We are grateful to Drs. C. G Extavour and M. Akam, University of Cambridge, for the Vasa antibody. We also thank members of the Shimizu laboratory for advice and help in collecting embryos. This study was supported in part by a Grant-in-Aid from the Ministry of Education, Science, Sports and Culture, Japan (13680799) to T.S.

\section{References}

Braat A, Zandbergen T, van de Water S, Goos H, Zivkovic D (1999) Characterization of zebrafish primordial germ cells: morphology and early distribution of vasa RNA. Dev Dyn 216: 153-167

Brusca RC, Brusca G J (2003) Invertebrates, 2nd ed. S Sinauer, Sunderland.

Chang CC, Dearden P, Akam M. (2002) Germ line development in the grasshopper Schistocerca gregaria: vasa as a marker. Dev Biol 252: 100-118

Dearden P, Grbic M, Donly C (2003) Vasa expression and germ-cell specification in the spaider mite Tetranychus urticae. Dev Genes Evol 212: 599-603

Dixon GC (1915) Tubifex. In: Herdman, W. A. (ed), L. M. B. C. Memoirs on Typical British Marine Plants and Animals, vol. 23. Williams and Norgate, London, pp 1-100

Fabioux C, Huvet A, Lelong C, Robert R, Pouvreau S, Daniel JY, Minguant C, Le Pennec M (2004) Oyster vasa-like gene as a marker of the germline cell development in Crassostrea gigas. Biochem Biophys Res Commun 320 592-598

Extavour CG (2005) The fate of isolated blastomeres with respect to germ cell formation in the amphipod crustacean Parhyale hawaiensis. Dev Biol 277: 387-402 
Extavour CG, Akam, ME (2003) Mechanisms of germ cell specification across the metazoans: epigenesis and preformation. Development 130: 5869-5884

Extavour CG, Pang K, Matus DQ, Martindale MQ (2005) vasa and nanos expression pattern in a sea anemone and the evolution of bilaterian germ cell specification mechanisms. Evol Dev 7: 201-215

Fujimoto T, Kataoka T, Sakao S, Saito T, Yamaha E, Arai K (2006) Developmental stages and germ cell lineage of the loach (Misgurnus anguillicaudatus). Zool Sci 23: 977-989

Fujimura M. Takamura K (2000) Characterization of an ascidian DEAD-box gene, $C i-D E A D 1$ : specific expression in the germ cells and its mRNA localization in the posterior-most blastomeres in early embryos. Dev Genes Evol 210: 64-72

Goto A, Kitamura K, Arai A, Shimizu T (1999a) Cell fate analysis of teloblasts in the Tubifex embryo by intracellular injection of HRP. Dev Growth Differ 41: 703-713

Goto A, Kitamura K, Shimizu T (1999b) Cell lineage analysis of pattern formation in the Tubifex embryo. I. Segmentation in the mesoderm. Int J Dev Biol 43: 317-327

Jamieson BGM (2006) Non-leech Clitellata. In: Rouse G, Pleijel F (eds) Reproductive Biology and Phylogeny of Annelida. Science Publishers, Enfield, pp 235-392

Kang D, Pilon M, Weisblat DA (2002) Maternal and zygotic expression of a nanos-class gene in the leech Helobdella robusta: primordial germ cells arise from segmental mesoderm. Dev Biol 245: 28-41

Kitamura K, Shimizu T (2000a) Embryonic expression of alkaline phosphatase activity in the oligochaete annelid Tubifex. Invert Reprod Dev 37: 69-73

Kitamura K, Shimizu T (2000b) Analyses of segment-specific expression of alkaline phosphatase activity in the mesoderm of the oligochaete annelid Tubifex: implications for specification of segmental identity. Dev Biol 219: 214-223

Knaut H, Pelegri F., Bohmann K, Schwarz H, Nüsslein-Volhard C (2000) Zebrafish vasa RNA but not its protein is a component of the germ plasm and segregates asymmetrically before germline specification. J Cell Biol 149: 875-888

Krфvel AV, Olsen LC (2002) Expression of a vas::EGFP transgene in primordial germ cells of the zebrafish. Mech Dev 116: 141-150

Lambert JD, Nagy LM (2002) Asymmetric inheritance of centrosomally localized 
mRNAs during embryonic cleavages. Nature 420: 682-686

Lasko P, Ashburner M (1988) The product of the Drosophila gene vasa is very similar to eukaryotic initiation factor-4A. Nature 335: 611-617

Mann KH (1962) Leeches (Hirudinea). Pergamon Press, Oxford

Matsuo K, Shimizu T (2006) Embryonic expression of a decapentaplegic gene in the oligochaete annelid Tubifex tubifex. Gene Expr Patterns 6: 800-806

Matsuo K, Yoshida H, Shimizu T (2005) Differential expression of caudal and dorsal genes in the teloblast lineages of the oligochaete annelid Tubifex tubifex. Dev Genes Evol 215: 238-247

Meyer A (1929) Die Entwicklung der Nephridien und Gonoblasten bei Tubifex rivulorum Lam. nebst Bemerkungen zum naturlichen System der Oligochäten. Z Z Wiss Zool 133: 517-562

Meyer A (1931) Cytologische Studien über die Gonoblasten und andere ähnliche Zellen in der Entwicking von Tubifex. Z Z Morph Oekol Tiere 22: 269-286

Nakamoto A, Arai A, Shimizu T (2000) Cell lineage analysis of pattern formation in the Tubifex embryo. II. Segmentation in the ectoderm. Int J Dev Biol 44: 797-805

Nakamoto A, Arai A, Shimizu T (2004) Specification of polarity of teloblastogenesis in the oligochaete annelid Tubifex: cellular basis for bilateral symmetry in the ectoderm. Dev Biol 272: 248-261

Nakao H (1999) Isolation and characterization of a Bombyx vasa-like gene. Dev Genes Evol 209: 312-316

Otani S, Maegawa S, Inoue K, Arai K, Yamaha E (2002) The germ cell lineage identified by vas-mRNA during the embryogenesis in goldfish. Zool Sci 19: 519-526

Pelegri F, Knaut H, Maischein H-M, Schulte-Merker S, Nüsslein-Volhard C (1999) A mutation in the zebrafish maternal-effect gene nebel affects furrow formation and vasa RNA localization. Cur Biol 9: 1431-1440

Penners A, Stäblein A (1930) Über die Urkeimzellen bei Tubificiden (Tubifex rivulorum Lam. und Limnodrilus udekemianus Claparede). Z Wiss Zool 137: 606-626

Pokrywka NJ (1995) RNA localization and the cytoskeleton in Drosophila oocytes. In: Capco DG (ed) Cytoskeletal Mechanisms during Animal Development. Academic Press, San Diego, pp 139-166

Rebscher N, Zelada-Gonzalez F, Banisch TU, Raible F, Arendt D (2007) Vasa unveils a 
common origin of germ cells and of somatic stem cells from the posterior growth zone in the polychaete Platynereis dumerilii. Dev Biol, doi:

10.1016/j.ydbio.2007.03.521

Sagawa K, Yamagata H, Shiga Y (2005) Exploring embryonic germ line development in the water flea, Daphnia magna, by zinc-finger-containing VASA as a marker. Gene Expr Patterns 5: 669-678

Schröder R (2006) vasa mRNA accumulates at the posterior pole during blastoderm formation in the flour beetle Tribolium castaneum. Dev Genes Evol 216: 277-283

Shinomiya A, Tanaka M, Kobayashi T, Nagahama Y, Hamaguchi S (2000) The vasa-like gene, olvas, identifies the migration path of primordial germ cells during embryonic body formation stage in the medaka, Oryzias latipes. Dev Growth Differ 42: 317-326

Shimizu T (1982) Development in the freshwater oligochaete Tubifex. In: Harrison FW, Cowden RR (eds) Developmental Biology of Freshwater Invertebrates. Alan R Liss, New York, pp 283-316

Shimizu T (1993) Cleavage asynchrony in the Tubifex embryo: involvement of cytoplasmic and nucleus-associated factors. Dev Biol 157: 191-204

Shimizu T (1995) Role of the cytoskeleton in the generation of spatial patterns in Tubifex eggs. In: Capco DG (ed) Cytoskeletal Mechanisms during Animal Development. Academic Press, San Diego, pp 197-235

Shimizu T (1996) Behaviour of centrosomes in early Tubifex embryos: asymmetric segregation and mitotic cycle-dependent duplicaton. Roux's Arch Dev Biol 205: 290-299

Shimizu T, Savage RM (2002) Expression of hunchback protein in a subset of ectodermal teloblasts of the oligochaete annelid Tubifex. Dev Genes Evol 212: 520-525

Shirae-Kuribayashi M, Nishikata T, Takamura K, Tanaka KJ, Nakamoto C, Nakamura A (2006) Dynamic redistribution of vasa homolog and exclusionof somatic cell determinants during germ cell specification Ciona intestinalis. Development 133: 2683-2693

Yisraeli J, Sokol S, Melton D (1990) A two-step model for the localization of maternal mRNA in Xenopus oocytes: involvement of microtubules and microfilaments in 
the translocation and anchoring of Vg1 mRNA. Development 108: 289-298

Yoon C, Kawakami K, Hopkins N (1997) Zebrafish vasa homologue RNA is localized to the cleavage planes of 2- and 4-cell-stage embryos and is expressed in the primordial germ cells. Development 124: 3157-3165

Yoshida-Noro C, Myohara M, Kobari F, Tochinai S (2000) Nervous system dynamics during fragmentation and regeneration in Enchytraeus japonensis (Oligochaeta, Annelida). Dev Genes Evol 210: 311-319

Wolke U, Weidinger G, Köprunner M, Raz E (2002) Multiple levels of posttranscriptional control lead to germ line-specific gene expression in the zebrafish. Cur Biol 12: 289-294

Zhou Y, King ML (1996) RNA transport to the vegetal cortex of Xenopus oocytes. Dev Biol 179: 173-183 


\section{Figure legends}

Fig. 1 a-r Summary of Tubifex development. a-q Selected stages of embryonic development. a-e Animal pole views of embryos at stages 1-cell (a), 2-cell (b), 4-cell (c), 8-cell (d) and 10-cell (e). f Stage 8 embryo. Posterior view with dorsal to the top. g Stage 11 embryo with ectoteloblast precursors (NOPQI, NOPQr), mesoteloblasts $(M l, M r)$ and endodermal precursors $\left(E^{D}\right)$. $\quad$ h Stage 12c embryo at the completion of teloblastogenesis. Dorsal view with anterior to the top. i-k Left side (upper) and ventral (lower) views of embryos undergoing gastrulation. mc micromere cap. l-q Left side views of elongating embryos. Asterisks indicate stomodaeum. pr prostomium. l-n Stage 16 embryos undergoing body elongation in their anterior half. Early, mid and late portions of stage 16 are designated stages 16a (l), 16b (m), and 16c (n), respectively. $\quad$ o-p Stage 17 embryos undergoing body elongation in their posterior half. Stage 17 is subdivided into stage $17 \mathrm{a}(\mathbf{o})$ and stage $17 \mathrm{~b}(\mathbf{p})$. $\quad \mathbf{q}$ Stage 18 embryo at completion of embryogenesis (and at the beginning of juvenile stage). $\mathbf{r}$ Time course of Tubifex development (stages 1 to Juv-D1 at $22^{\circ} \mathrm{C}$ ). Juv-D1 1-day-old juvenile.

Fig. 2 Temporal expression profile of Ttu-vas. RT-PCR analysis showing expression pattern of Ttu-vas transcripts in T. tubifex embryos from stage 1 to stage 18 . For details of developmental stages, see Fig. 1 and Shimizu 1982. $\quad \beta$-Actin was used as positive control.

Fig. 3 a-m Expression of Ttu-vas during early cleavage. All embryos except a, b were cleared and viewed with transmitting light. pp pole plasm. a-b Animal (a) and vegetal (b) pole views of egg at $1 \mathrm{~h}$ after the second meiosis. Uncleared specimen. Asterisks indicate the poles. c Animal pole view of another egg at $1 \mathrm{~h}$ after the second meiosis. Arrow indicates the nuclear region. d Animal pole view of egg at anaphase of the first mitosis. Ttu-vas RNA is concentrated in the mitotic spindle. Arrowhead and double arrowhead indicate astral spindle pole and anastral pole, respectively. The dark stain seen in the region corresponding to the midzone of the spindle associates with concentrated pole plasms. e-f Stage 2 embryos with CD cell at metaphase of the second mitosis. $\mathbf{f}$ is an enlargement of $\mathbf{e}$. Arrows indicate the spindle poles. $\mathbf{g}$ Nocodazole-treated 2-cell embryo. Animal pole view. This embryo was treated with 
$5 \mu \mathrm{g} / \mathrm{ml}$ nocodazole for $1 \mathrm{~h}$ before fixation. Ttu-vas RNA is distributed in pole plasm (pp) and cortex (arrow). h Stage 4 embryo. Animal pole view. Arrows indicate mitotic spindles in cells C and D. i Stage 8 embryo shortly after division of 3D into 4d and 4D. Right side view. Arrows indicate asters in $2 \mathrm{~d}^{11}$; arrowhead mitotic spindle in 3C; double arrowhead nuclear region of 4D cell. $\mathbf{j}$-l Late stage 8 embryo.

Posterior view. $\quad \mathbf{k}$ and $\mathbf{l}$ are enlargements of $\mathbf{j}$. Arrows indicate spindle poles. $\mathbf{m}$ Early stage 11 embryo. Posterior view with dorsal to the top. M teloblasts are undergoing cell division to form first primary m-blast cells (arrowheads). Arrows indicate spindle poles in endodermal cells $\left(E^{D}\right)$ derived from $4 \mathrm{D}$ cell. Scale bar in a indicates $200 \mu \mathrm{m}$ (for a-e, g-j, m) and $100 \mu \mathrm{m}$ (for $\mathbf{f}, \mathbf{k}, \mathbf{l}$ ).

Fig. 4 a-i Expression of Ttu-vas during teloblastogenesis. All embryos except a, b, $\mathbf{g}$ were cleared and viewed with transmitted light. a-d Late stage 11 embryos. Asterisks indicate a "cap”, comprised of a subset of micromeres. a-b Two different views of the same embryo. Uncleared specimen. c Arrows indicate mitotic spindle in NOPQ cell. d Posterior view showing primary m-blast cells (arrowheads) and M teloblasts. e-f Stage 12a embryos after division of NOPQ into N and OPQ. Right side view with dorsal to the top. $\mathbf{f}$ is an enlargement of $\mathbf{e}$. Incipient ectodermal (arrowheads) and mesodermal (double arrowheads) germ bands consist of Ttu-vas-expressing cells. Arrows indicate mitotic spindles in OPQ cell and M teloblast. g-i Stage $12 b$ embryos prior to division of OP cell. $\mathbf{g}$ Right side view with dorsal to the top. Uncleared specimen. Note a band of Ttu-vas-expressing cells extending from the arc of cells N, OP and Q. h-i Dorsoposterior views in different focal planes. Arrowheads indicate incipient ectodermal germ band. Arrows indicate mitotic spindles. Scale bar in a indicates $200 \mu \mathrm{m}$ (for a-e, g-i) and $100 \mu \mathrm{m}$ (for f).

Fig. 5 a-m Occurrence of ventral Ttu-vas-expressing cells during gastrulation in normal embryos (a-h) and actinomycin D-treated embryos (i-m). a-f, g, h, left side views with dorsal to the top; b, d, f, ventral views. a-b Stage 14 embryo. c-d Stage 15 embryo. $\quad$ e-h Stage 16a embryos. $\mathbf{h}$ is an enlargement of $\mathbf{g}$ (cleared specimen). Ttu-vas RNA is detected in germ bands $(\mathrm{gb})$ at stages 14 and 15 (a-d), but not at stage 16a (e, f) except large cells aligning along the ventral midline (e-h). (i-m) Embryos at the beginning of stage 13 were treated with $200 \mu \mathrm{g} / \mathrm{ml}$ actinomycin $\mathrm{D}$, fixed $48 \mathrm{~h}$ later, 
and processed for in situ hybridization. $\quad \mathbf{j}-\mathbf{m}$ show cleared specimens. $\quad \mathbf{i}-\mathbf{j}$ show the same embryo; $\mathbf{k}$ and $\mathbf{m}$ are enlargements of $\mathbf{j}$ and $\mathbf{l}$, respectively. In all panels, anterior is to the left. Arrows indicate Ttu-vas-expressing cells. Scale bar in a indicates 200 $\mu \mathrm{m}$ (for a-g, $\mathbf{i}, \mathbf{j}, \mathbf{l}$ ) and $100 \mu \mathrm{m}$ (for h, $\mathbf{k}, \mathbf{m}$ ).

Fig. 6 a-l Patterns of Ttu-vas-expressing cell distribution along the anteroposterior axis in stage 16a embryos. Ventral views with anterior to the top. A pair of asterisks in each panel indicate the boundary between segments IX and X. Numerals at the upper right of each panel indicate the frequency (in percentage) of each pattern $(n=60)$. Scale bar in $\mathbf{l}$ indicates $200 \mu \mathrm{m}$ (for a-l).

Fig. 7 a-k Frequency of Ttu-vas-expressing cell occurrence with reference to body segments. Abscissa: frequency (in percentage) of cell occurrence. Data from the left side and the right side of the embryo are presented separately. Ordinate: position of segments (V to XIII). For stages 16a to 18, see Fig. 1. Juv-D1, D2, D3, D4, and D5 are referred to stages for juveniles, respectively, at 1, 2, 3, 4 and 5 days after completion of embryogenesis. Asterisks in $\mathbf{h}$-k indicate the occurrence of Ttu-vas-positive tiny dots (see Fig. 9c-e).

Fig. 8 a-e Ventral Ttu-vas-expressing cells in juveniles. a-d 2-day-old juveniles (Juv-D2); e 3-day-old juvenile (Juv-D3). In all panels, anterior is to the left. a, e left side view; b-d ventral view. Ttu-vas-expressing cells are situated in the anterior margin of segments X and XI. Arrows in c-e indicate Ttu-vas-positive tiny dots. Scale bar in a indicates $200 \mu \mathrm{m}$ (for a-e) and $1 \mathrm{~mm}$ (for insets).

Fig. 9 a-h Expression of Ttu-vas protein in Tubifex embryos (a-g) and juvenile (h). Embryos in a-g were all cleared for observation after immunostaining; the juvenile in $\mathbf{h}$ was uncleared. a 1-cell embryo (at $1 \mathrm{hr}$ after the second meiosis) and stage 6 embryo with cells $2 \mathrm{~d}$ and 2D. Ttu-vas protein is abundant in pole plasms ( $p p)$ and nuclei (arrowheads). b-c Late stage 12 embryo. Dorsal view with anterior to the top. $\quad \mathbf{c}$ is an enlargement of $\mathbf{b}$. Ttu-vas protein is concentrated in perinuclear region (arrowheads) in nearly all of the blastomeres. Mesoteloblasts $(M)$ appear to contain more Ttu-vas protein than any other blastomeres. d-g Stage 16c (d, e) and 17a (f, g) 
embryos. Left side views with dorsal to the top. Two focal planes are shown to depict Ttu-Vas-positive cells on the left side $(\mathbf{d}, \mathbf{f})$ and the right side $(\mathbf{e}, \mathbf{g})$ of each embryo. Horizontal lines indicate positions of segments with Ttu-Vas-positive cells. The inset shows higher magnification of positive cells in segments X to XII seen in $\mathbf{f}$. h 5-day-old juvenile (Juv-D5). Right side view; asterisk indicates the anterior end. Arrows indicate Ttu-Vas-positive cells in segments X and XI. Scale bar in h indicates $200 \mu \mathrm{m}$ (for a-h); $100 \mu \mathrm{m}$ (for inset).

Fig. 10 Occurrence of ventral Ttu-vas-expressing cells in embryos deprived of cells $2 \mathrm{~d}^{111}$ and $4 \mathrm{~d}$. Ventral views with anterior to the top. Either anteroposterior or dorsoventral polarity is obscure in embryos shown in $\mathbf{c}$ and $\mathbf{d}$. a-b Control embryos that had been cultured without vitelline membrane for 5 (a) or 10 (b) days after stage 8. Ttu-vas-expressing cells occurred in a normal fashion. Segments X and XI are indicated by vertical lines. c-d Stage 8 embryos were deprived of $4 \mathrm{~d}$ cell, and cultured for 5 (c) or 10 (d) days before fixation for in situ hybridization. At any time point of development, there were no trace of Ttu-vas-expressing cells. Arrows indicate cell clumps derived from ectoteloblasts. e-f Stage 8 embryos were deprived of $2 \mathrm{~d}^{111}$ cell, and cultured for 5 (e) or 10 (f) days before fixation. These embryos had undergone body elongation to some extent (see upper inset in $\mathbf{f}$ ). Arrows indicate Ttu-vas-expressing cells. Lower inset in $\mathbf{f}$ shows left side view of Ttu-vas-expressing cells (arrows). Scale bar in a indicates $200 \mu \mathrm{m}$ (for a-f) and $1 \mathrm{~mm}$ (for insets).

Fig. S1 Characterization of Ttu-vas, a vasa homologue from T. tubifex. a Alignment of Ttu-Vas with known Vasa-class proteins. The eight conserved motifs of the DEAD-box protein family are boxed in black. The EARKF and WD motifs are boxed in red. Numbers in parentheses indicate the percentage amino acid identity with the overall sequences of Ttu-Vas. b Molecular phylogenetic relationship of Ttu-Vas to other DEAD-box proteins. The phylogenetic tree was generated by the neighbor joining method using PAUP*4.0b10. Dme-Abs was used as an outgroup. Numbers are bootstrap values (as percentages of 1000 replications). Lengths of branches are drawn to the scale indicated (Bmo Bombyx mori, Cgi Crassostrea gigas, Csa Ciona savignyi, Dma Daphnia magna Dme Drosophila melanogaster Dre Danio rerio, Gga Gallus gallus, Hma Hydra magnipapillata, Mmu Mus musculus, Ola Oryzias latipes, 
Pdu Platynereis dumerilii, Ttu Tubifex tubifex).

Fig. S2 Frequency of total number of ventral Ttu-vas-expressing cells present on the left (a) and right (b) side of individual embryos. Abscissa: developmental stages (16a to 18) and juvenile stages (Juv-D1 to D3). 


\begin{tabular}{|c|c|c|c|c|c|c|c|c|c|c|c|c|}
\hline stages & 1 & $2-4$ & 5 & $6-7$ & 8 & $9-10$ & 11 & 12 & $13-15$ & 16 & 17 & 18 \\
\hline tu-vas & 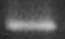 & 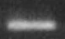 & $=$ & 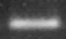 & 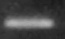 & $=$ & $=$ & 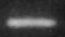 & -2 & & & \\
\hline$\beta$-actin & $\lim 4$ & nese & $\min$ & 2 & enses & chases & 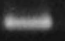 & 8 & $\cos$ & -6 & cis & \\
\hline
\end{tabular}




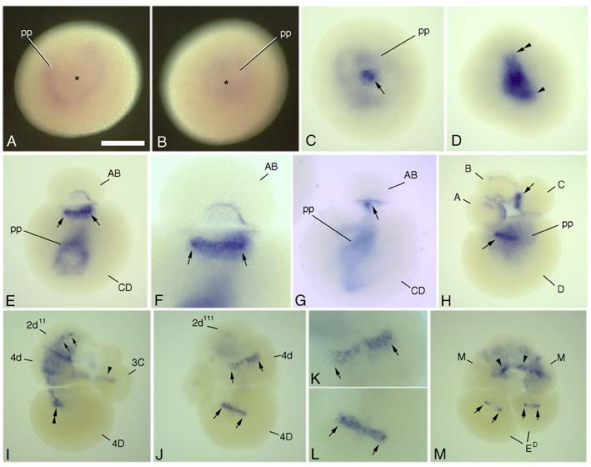




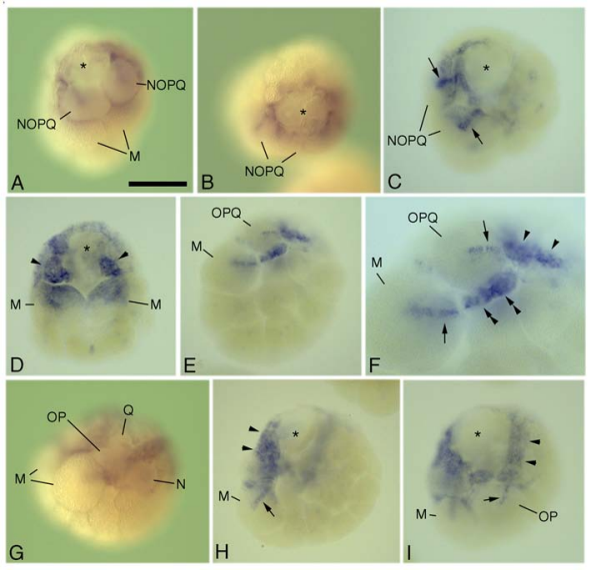




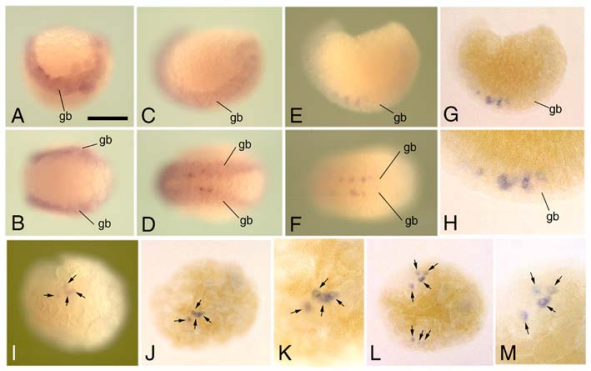




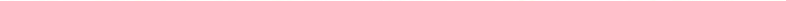




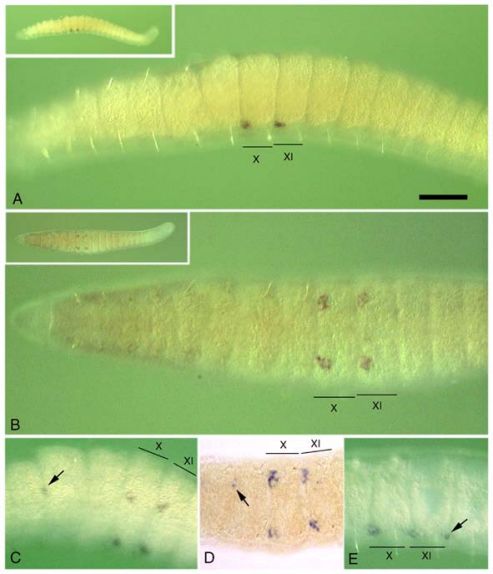




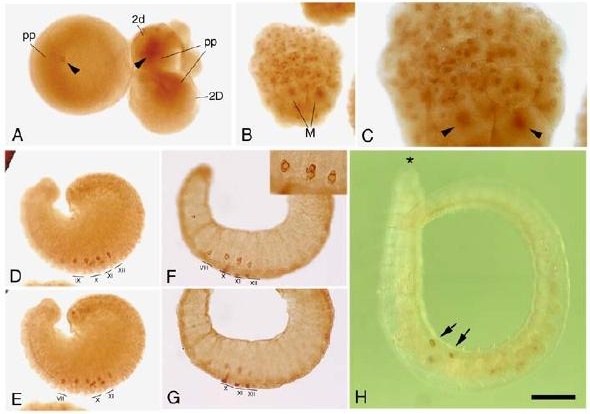




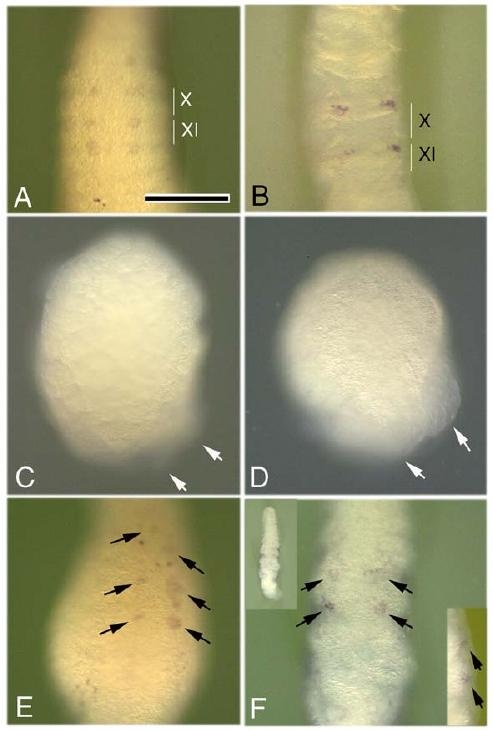


Theves

pusura

Cgs-Ves

Oterves

Der-Ves

Thus Was

Pelyevas

Cot-ins.

Quevios

ree-Wos

Teyslas

padarvas

Cgi-Wes

Olawhos

Den-Ves.

tru-tes

Piveles

C;1-ines

0lentes

Dnt-ies

Tueves

pd-Ves

cathes

Olo-kes

one-tas

Itw-ics

Pon-les

Cas-Mes

gie-Nes

Dremba

Itw-ins

for les

Castics

Diquest

Dereses

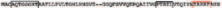

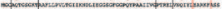

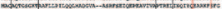

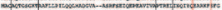

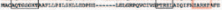

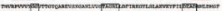

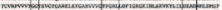

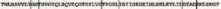

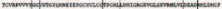

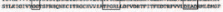

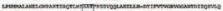

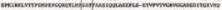

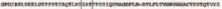

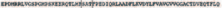

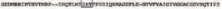

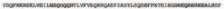

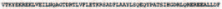

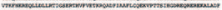

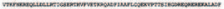

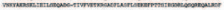

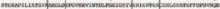

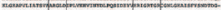

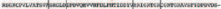

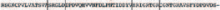

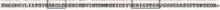

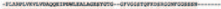

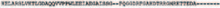

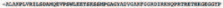

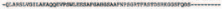

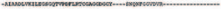

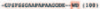

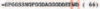

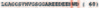

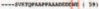

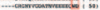

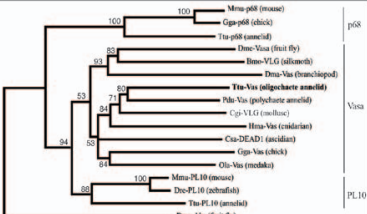




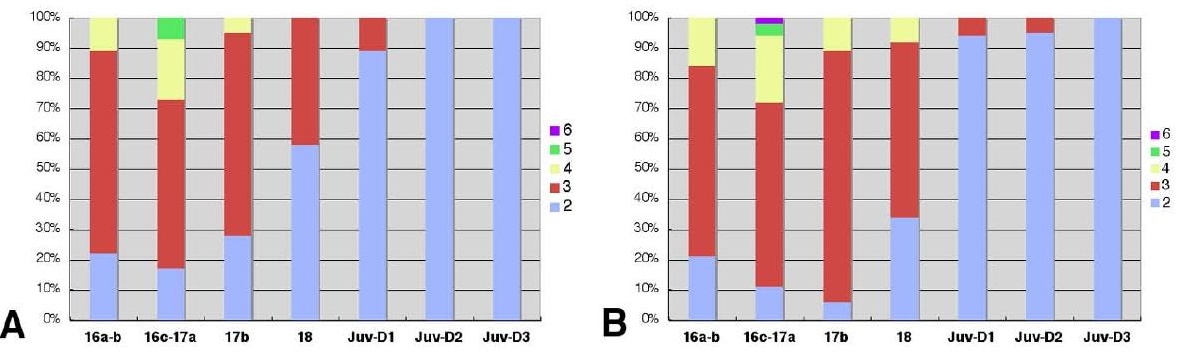

